\title{
Evaluation of articular disc loading in the temporomandibular joints after prosthetic and pharmacological treatment in model studies
}

\author{
Małgorzata E. Pihut ${ }^{1, A, B, D}$, Jerzy Margielewicz ${ }^{2, C}$, Edward Kijak ${ }^{3, E}$, Grażyna Wiśniewska ${ }^{1, F}$ \\ ${ }^{1}$ Department of Prosthodontics, Jagiellonian University Medical College, Kraków, Poland \\ 2 Department of Logistics and Mechanical Handling, Faculty of Transport, Silesian University of Technology, Katowice, Poland \\ ${ }^{3}$ Department of Prosthodontics, Pomeranian Medical University, Szczecin, Poland \\ A - research concept and design; B - collection and/or assembly of data; C - data analysis and interpretation; \\ $D$ - writing the article; $E$ - critical revision of the article; $F$ - final approval of article
}

Address for correspondence

Małgorzata Pihut

E-mail: pihut_m@poczta.onet.pl

Funding sources

None declared

Conflict of interest

None declared

Received on October 31, 2015

Revised on February 28, 2016

Accepted on March 16, 2016

DOI

$10.17219 /$ acem $/ 62216$

\section{Copyright}

Copyright by Author(s)

This is an article distributed under the terms of the

Creative Commons Attribution Non-Commercial License

(http://creativecommons.org/licenses/by-nc-nd/4.0/)

\begin{abstract}
Background. Temporomandibular joint dysfunction is often related to excessive load in the stomatognathic system.

Objectives. The objective of the model tests, using numeric calculations, was to assess the articular disc loads in the temporomandibular joints after prosthetic and pharmacological treatment of functional disorders of the masticatory organ.
\end{abstract}

Material and methods. The study involved 10 patients, aged 21-48 years, of both sexes, randomly selected from a group of 120 patients treated with relaxation occlusal splints (60 patients, group I) and intramuscular injection of botulinum toxin type A (60 patients, group II), suffering from temporomandibular joint dysfunction with the dominant muscle component. In all subjects, a specialized functional examination was carried out. Treatment groups: occlusal splint therapy (group I) and intramuscular injection of botulinum toxin type A (group II). An assessment of the loads of 4 disc zones of the temporomandibular joints was carried out based on the results of clinical studies (phase I of the study), and numeric model tests (phase II). In the representatives of the study groups (5 patients in each group), measurements of occlusal forces and an evaluation of tension of the masseter and temporalis muscle were performed.

Results. The results of the average load values for all evaluated zones of the right and left articular disc differ in a statistically significant way in favor of group $I I$, with the exception of the external mid part of the discs. In the case of the anterior of the right disc, the load was lower in patients belonging to group I than in those obtained in group $\|$.

Conclusions. Botulinum toxin type A significantly reduces the loads within the temporomandibular joints, generated by masseter muscle hypertonia.

Key words: articular disk loading, temporomandibular joint dysfunction, botulinum toxin type A, prosthodontics treatment 
Temporomandibular joint dysfunctions, according to WHO reports, are the third of dental diseases in prevalence following dental caries and periodontal diseases. ${ }^{1}$ The type of these disorders is determined by genetic, individual, environmental and psycho-emotional factors. It has been observed that an increasing level of stress leads to growth in harmful movement habits within the stomatognathic system, and a rapid increase in muscle tension adversely affects the functioning of the temporomandibular joints. Under physiological conditions articular load is favorable, enabling the correct functioning of the structure, but in the situation when the load is too high, there is often significant surface and disc damage within the joints. ${ }^{2-15}$

The objectives of the model tests, using numeric calculations, was to assess the articular disc loads in the temporomandibular joints after prosthetic and pharmacological treatment of functional disorders of the masticatory organs.

The study involved 10 patients, aged $21-48$ years, of both sexes, with full dental arches, randomly selected from a group of 120 patients treated with relaxation occlusal splints (60 patients - group I) and intramuscular injection of botulinum toxin type A (60 patients - group II). The group of 10 representative patients were created from 5 patients taken from group I and 5 from group II.

In all the patients, a specialized functional diagnostic procedure showed the presence of temporomandibular joint dysfunction with the dominant muscle component. The effects of excessive tension of the masticatory muscles were observed as symptoms in teeth and periodontium as well. In the representative group of 10 patients, there were no painful forms of dysfunction. The treatment was performed in the Prosthodontics Clinic of the Jagiellonian University Medical College in Kraków.

In all subjects, a specialized functional examination was carried out, according to the application form developed in the Consulting Room of Temporomandibular Joint Dysfunction of the Prosthodontics Department of Jagiellonian University. The form is based on the typical routine requirements of a functional evaluation of the stomatognathic system, such as: a rating of the mandibular range of movement, symmetry of mandible motion, deviation of opening path, occlusion condition, masticatory muscle sensitivity during palpation, pain of their attachments, and the presence of acoustic and pain symptoms in temporomandibular joints occurring during mandibular movement and palpation examination. Additional examination was concerned with an evaluation of pain intensity and imaging diagnosis. ${ }^{1,2}$ The patients were qualified for 2 prosthetic treatment groups: occlusal splint therapy (group I) and intramuscular injection of botulinum toxin type A with a total amount of $21 \mathrm{MU}$ (mouse units) for each muscle (group II). The injections were done at the 3 points of vertices of a triangle ( 7 units in each point) along the largest cross-sectional of the muscle, 1 time, using a $40 \mathrm{U}$ insulin syringe. The pharmacological activity of the toxin lasts about 12 weeks. ${ }^{1,6}$

The functional examination of the masticatory organ, masseter muscles and temporomandibular joint pain intensity and masseter muscle electrical activity were evaluated 4 times: before the prosthetic treatment began, after 10 days of the initiation of the 2 different treatment methods (splint and intramuscular injections) and after 14 and 22 weeks from the second examination.

An assessment of the loads of 4 disc zones of the temporomandibular joints was carried out based on the results of clinical studies (phase I of the project), and numeric model tests (phase II). ${ }^{1,17,18}$ In the representatives of the study groups (5 patients in each group), measurements of occlusal forces were performed using the method developed by professor W. Chladek as well as an evaluation of the masseter muscle tension and the anterior temporal muscles using a Bio Research EMG I and surface electrodes. ${ }^{10,17}$

The maximum occlusal forces were evaluated around the central incisors and the first molars of the left and right side, using a special measuring device - developed for the needs of dental research in the Department of Mechanical Technology and Forming of Silesian Technical University in Katowice (patent no. P 334933). In order to calculate the maximum values of occlusal forces using the above method, the Mayer's formula was applied:

$$
F=c \cdot d^{n}
$$

where: $\mathrm{c}$ - a material constant; $\mathrm{d}$ - diameter of dents in the aluminum sample; $\mathrm{n}$ - Mayer's coefficient - 1.706 .

The constant " $c$ " and coefficient " $n$ " appearing in the formula were specified for the series of aluminum samples applied in the research by measuring the diameters of the indentations formed during the calibration of the instrument in the universal testing machine. This formula determines the correlation of the resulting indentation size to pressure-gradient force penetrator, and hence the tooth pressure force on the measuring device. To measure the diameter of the dents in the disposable aluminum plates, a stereoscopic measuring microscope was used. ${ }^{10,11,18}$

Since the direct measurement of loads in the temporomandibular joints in a living human is not possible, a computer model of the mandible, projecting masticatory organ functions, was constructed, which uses the following model assumptions:

1. For numeric calculations, the mandible was considered a non-deformable body, since the intended purpose of the study is not to evaluate the size of deformations of the mandible.

2. The studies assumed the average orientations of muscle forces, due to the fact that the muscle attachments are located in particular areas of the jaw or skull bones and can be "reduced" to the points in the model study, because the results of the clinical studies carried out on 
various types of face - long and short - have shown the convergence of the lines of action of the muscle force vectors in the case of both types of face.

3. The loads occurring in the temporomandibular joints have been identified on the basis of a spatial model of the human masticatory system according to Margielewicz's concept.

4. Forces exerted on the temporomandibular joints were estimated based on the load acting on the articular discs. In the numeric calculations the replacement stiffness of discs was taken into account as the actual nonlinear displacement-force characteristics make it difficult to perform numeric calculations.

5. The resilient properties of the muscles were projected using mechanical two-terminals, because the data characterizing the volumes of the cross-section of the muscle, its rest length, and the unit capacity are available.

Analytical dependency mapping replacement stiffness of the masticatory muscle fiber groups was calculated according to the following formula:

$$
c_{z}=\frac{k A}{l}
$$

where: $\mathrm{k}$ - the coefficient of unit performance of the cross section of the muscle equals $40\left[\mathrm{~N} / \mathrm{cm}^{2}\right]$; A - the maximum cross-section of the muscle; $\mathrm{L}$ - the rest length of the muscle. ${ }^{16,17}$

From the point of view of biomechanics, one of the most important pieces of information about the masticatory organ muscles is the maximum forces generated by individual masseter muscles. They refer to the largest cross-sections of the muscles, therefore an important element for a model study of the stomatognathic system is the so-called coefficient of the unit performance of the cross section of the muscle. The value of this coefficient, according to Chladek, is $40-100 \mathrm{~N} / \mathrm{cm} .{ }^{2,10}$

Proportions occurring between the maximum (limit) muscle forces and their bioelectric activity were calculated according to the formula:

$$
\frac{E M G_{M P}}{F_{M P}}=\frac{E M G_{M G}}{F_{M G}}
$$

where: $\mathrm{EMG}_{\mathrm{MP}}$ - bioelectric activity of the surface fibers of the masticatory organ, $\mathrm{EMG}_{\mathrm{MG}}$ - bioelectric activity of the medial pterygoid, $\mathrm{F}_{\mathrm{MP}}$ - the maximum force generated by the surface fibers of the masticatory organ, $F_{M G}$ - the maximum force generated by the medial pterygoid.

For the purposes of this study, the results of maximum occlusal forces, the electrical potential values of masseter and temporal muscles were discussed as well as the model tests following the prosthetic treatment carried out with the use of the two compared methods.

In the case of the clinical measures giving constant results, statistical analysis was based on the traditional methods of calculation: mean values, standard deviation, minimal values, maximal values, standard error of the mean, variance analysis for dependent variables and posthoc Tukey test for dependent variables being the statistical significance measure.

To compare the dependencies between the clinical results obtained in consecutive clinical tests, the non-parametric Friedman test, Kendall's W and Wilcoxon signedrank test (comparing 2 related samples) were used. For the statistical studies, special STATISTICA 2010 computer software was used.

Only a few of the clinical studies were used in numeric model tests. The results of the disc loads in the temporomandibular joints in numeric model tests on the representatives of the study groups were statistically analyzed using the parametric Student's t-test and it was assumed that the results were statistically significant if $\mathrm{p} \leq 0.05$.

\section{Results}

In the numeric model test, projecting the stomatognathic system activity, carried out on the representatives of the 2 groups, a significant difference in articular disc loads was observed between patients undergoing the splint therapy and botulinum toxin type A in favor of the second group.

The results of the clinical research of maximal bite forces (5 patients in group I and II) are presented in Fig. 1 and they were the basis for the work on the numeric tests. This was necessary for identification of the loads within the temporomandibular joints. Table 1 shows the results of the electromyographic measurements obtained in patients randomly selected from both groups evaluated for model tests in order to calculate the loads of the temporomandibular joints after the therapy using occlusal splints and after the application of botulinum toxin type A. The average values of the electrical potentials obtained during the clinical study of the right and left masseter and temporalis muscles were important to create the numerical model of a biocybernetic model of the masticatory system. The ranges of these values were $15.71-96.11 \mu \mathrm{V}$ for both types of muscles. In this individual study, the numeric values of the replacement coefficients of articular disc zone rigidity were applied, calculated by Chladek et al. ${ }^{10,11}$

The average values of loads (evaluated in newtons) acting on the individual disc zones of the left and right temporomandibular joint are summarized in Tables 2 and 3 . The results of the average load values for all the evaluated zones of the right and left articular disc differ in a statistically significant way in favor of group II, with the exception of the external mid part of the discs. In the case of the anterior of the right disc, the load was lower in patients belonging to group I than in those obtained in group II. 
Table 1. Average values of electrical potentials obtained during clinical studies in order to create the numeric model in microvolts $[\mu \mathrm{V}]$

\begin{tabular}{|c|c|c|c|c|c|}
\hline \multirow{2}{*}{\multicolumn{2}{|c|}{ Patient number }} & \multicolumn{2}{|c|}{$\begin{array}{c}\text { Masseter muscle } \\
\text { surface fibers, EMG } \\
\text { MP }\end{array}$} & \multicolumn{2}{|c|}{$\begin{array}{c}\text { Temporal muscle } \\
\text { anterior fibres, EMG }\end{array}$} \\
\hline & & right side & left side & right side & left side \\
\hline \multirow{5}{*}{ GI } & 1 & 45.21 & 35.77 & 40.38 & 55.31 \\
\hline & 2 & 113 & 85.22 & 96.11 & 114.57 \\
\hline & 3 & 84.21 & 45.21 & 54.93 & 73.15 \\
\hline & 4 & 21 & 15.71 & 21.88 & 39.07 \\
\hline & 5 & 45.31 & 59.24 & 61.73 & 73.98 \\
\hline \multirow{5}{*}{ GII } & 1 & 45.68 & 39.21 & 48.98 & 55.98 \\
\hline & 2 & 85.14 & 75.1 & 65.98 & 76.1 \\
\hline & 3 & 24.32 & 32.14 & 28.9 & 39.17 \\
\hline & 4 & 41.21 & 30.14 & 36.4 & 57.91 \\
\hline & 5 & 19.27 & 24.19 & 38.1 & 47.91 \\
\hline
\end{tabular}

Table 2. Average values of the forces acting on the right articular disc in $[\mathrm{N}]$ and statistical significance denoted by letter $\mathrm{p}$

\begin{tabular}{|l|c|c|c|}
\hline \multicolumn{1}{|l|}{ Articular disc zone } & Group I & Group II & p-value \\
\hline Anterior & 6.8 & 10.76 & 0.00001 \\
\hline Posterior & 119 & 123.7 & 0.00000 \\
\hline External mid & 59.2 & 47.9 & 0.06000 \\
\hline Internal mid & 62 & 63.5 & 0.000001 \\
\hline
\end{tabular}

Table 3. Average values of the forces acting on the left articular disc in [N] and statistical significance denoted by letter $p$

\begin{tabular}{|l|c|c|c|}
\hline \multicolumn{1}{|c|}{ Articular disc zone } & Group I & Group II & p-value \\
\hline Anterior & 23.52 & 15.14 & 0.000016 \\
\hline Posterior & 123.24 & 93.72 & 0.000011 \\
\hline External mid & 33.58 & 23.16 & 0.000021 \\
\hline Internal mid & 30.08 & 21.84 & 0.000010 \\
\hline
\end{tabular}

\section{Discussion}

The main aim of the treatment of temporomandibular joint dysfunction is the remission of pain of the muscles and joints and restoration of the physiological norm for proper muscle tension and load of the joint. For the proper functioning of the stomatognathic system, it is very important to provide physiological stress on joints.
It ensures proper distribution of synovial fluid and blood supply, which is necessary within the articular surface. ${ }^{1,3,4}$ In the course of functional disorders, the masticatory system comes to excessive and highly damaging overloads of the joints and intra-articular discs, which is caused by a significant increase in masseter muscle tension. . $^{3,7,10,12,13,18,19}$

Occlusal splints, pharmacological treatment in the form of intramuscular injection and muscle exercises are methods that have been shown to have a positive effect on the state of masticatory muscle relaxation, which can be confirmed by objective testing instruments such as electromyography and occlusal evaluation using a computer program, and T-Scan device. Botulinum toxin is produced by Gram-positive Clostridium bacteria. In their natural environment, these bacteria form spores that release the neurotoxin (the strongest known to man) when they germinate. There are 6 serotypes of the toxin: A, B, C, D, E, F, and G. Botulinum toxin type A was first isolated in 1920s. Its therapeutic potential was put to the test for the first time then. All 7 serotypes are large proteins that act on cholinergic neuromuscular junctions to block transmission of synaptic vesicles. ${ }^{1,15}$ Edward Schantz and Alan Scott were pioneers of the therapeutic applications of the botulinum toxin used from the late 1970s to manage strabismus and hemifacial spasms at first and then eyelid spasm, uterine atony, and laryngological, nephrological, surgical and neurological therapies as well as aesthetic medicine. The medication is currently registered in approx. 120 countries. ${ }^{15}$

An assessment of disc loading within the temporomandibular joints of patients is not possible. In the stomatognathic system, direct measurement of living human articular disc loads and surface TMJ is not possible, therefore construction of a special model, which was based on the concept of one of the authors of this study, is an important instrument in establishing the initial orientation of the articular disc load changes depending on the state of muscle tension, and has not been used in the assessment of the effectiveness of treatment of the masticatory system by other authors at all..$^{1,10,16-23}$

Despite the rapid growth in model testing and biotechnological studies, only a few reports in the literature assess the loads within the temporomandibular joints. The assessment of the relationship between bite forces and occlusion, and the activity of muscle and loads acting on the temporomandibular joints is a difficult issue from a biomechanics perspective. ${ }^{17,18}$

Results of the research available in the literature are mostly related to the evaluation of mechanical strain or anatomical changes in the properties of porcine discs, which are similar in structure to human discs. Experimental studies conducted on the intra-articular tissues derived from animals (cattle and pigs) for an assessment of the resistance to crushing or tensile strength have provided valuable information about the mechanical proper- 
Fig. 1. Average values of the occlusal forces measured in groups' representatives following the treatment

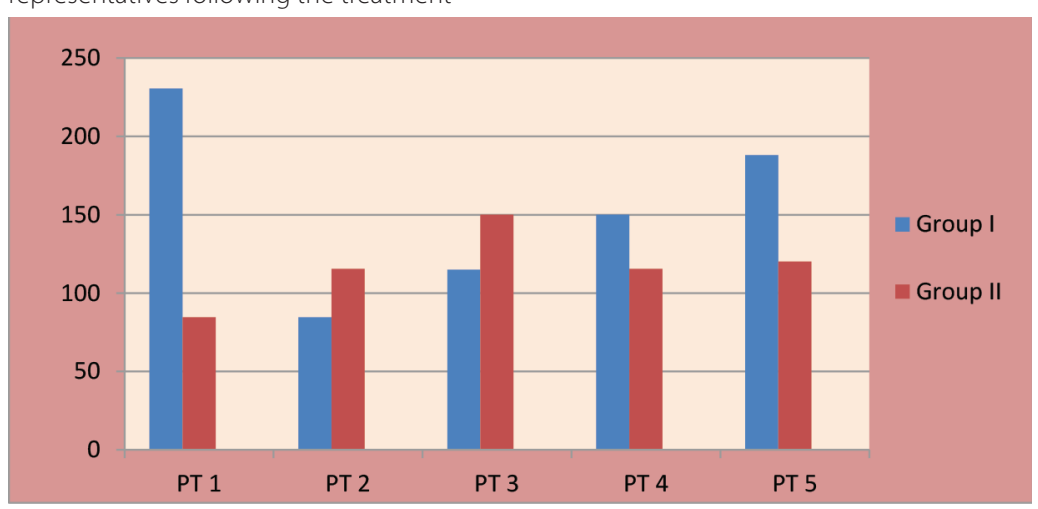

in the loads between the 2 groups was 27.79 $\mathrm{N}$ in favor of group II. The least significant load differences between groups were calculated for the right discs in the outer middle zone $(2.4 \mathrm{~N})$ and in the front zone. Chladek et al. notes that excessive static and dynamic TMJ load leads to rapid deformation of the articular disc and impairment of the flow of fluids in the disc and the zone outside the disc. These factors have a decisive influence on the development of dysfunctions within the joint system. ${ }^{10,11,28}$ Nickel et al., in the study carried out in 52 patients with the pain form of the functional disorders assessing the TMJ load, found that a decrease in

ties of these structures, however, any changes in the in vitro environment had an important impact on the final value obtained. ${ }^{10,11}$

Fernandez P. et al. conducted research of the dynamic compressive properties in 5 differentiations of the porcine temporomandibular joint disc which were investigated over a wide range of loading frequencies. The results of this investigation suggest that the dynamic viscoelastic compressive modulus is region-specific and depends on the loading frequency and has important implications for the transmission of load to the temporomandibular joints. ${ }^{25}$ Model tests were also performed by Commisso et al., who evaluated an algorithm for adjusting the quasi-liner viscoelastic model to fit a material using a stress relaxation test and validating a protocol for performing such tests in temporomandibular joint discs. ${ }^{26}$ Juran et al. assessed regional variation in the disc's shear load characteristics under a physiologically relevant load and to associate those mechanical findings with common clinical observations of disc fatigue and damage. The results of these studies suggest that the posterior region of the disc is the most often the zone in which fatigue occurs, what may lead to disc damage and perforation. ${ }^{27}$

The numeric model tests, projecting stomatognathic system activities, carried out in the framework of this project, on the group of representatives treated with occlusal splints and intramuscular injection of botulinum toxin type A, show a significant difference in articular disc loads, demonstrating the positive impact of treatment using the pharmacological method.

Analyzing the results of the numeric model tests, higher load values in all the examined zones of the right and left discs were observed in the group of patients treated with occlusion splints (I), compared to the results obtained in the group treated with the injection of botulinum toxin (II), with the exception of the front zone of the right disc. The largest load values were obtained in model tests for the rear areas of both discs, because these values amount to 119 (right) and $123.24 \mathrm{~N}$ (left) in group I and, respectively, to 123.7 and $93.72 \mathrm{~N}$ in group II. Comparing the mean values for both discs for the rear discs zone - the difference joint pain is closely related to reductions in the articular surfaces loads. ${ }^{19}$ Our results indicate lower loads occurring within the joints improve the clinical parameters of the mandible dynamics in the course of treatment with the botulinum toxin, as compared to the treatment with occlusal splints.

\section{Summary}

In the treatment of temporomandibular joint dysfunction, we use many diagnostic and therapeutic methods such as the use of occlusal splints, pharmacological treatment, physiotherapy and psychological support. ${ }^{1,5,6,12,14,20,23,29}$ The results of model tests indicate a more favorable impact using intramuscular injection of botulinum toxin type A on the load condition within the temporomandibular joint compared to the results obtained in patients with occlusal splint. The increase in loads beyond the physiological level within the joint structures is a factor in pain of high intensity and irreversible morphological changes of all its elements. ${ }^{1,11,16,17}$

\section{Conclusion}

Botulinum toxin type A significantly reduces the loads of the disc within the temporomandibular joints generated by masseter muscle hypertonia.

\section{References}

1. Pihut M. The effectiveness of the prosthetic and pharmacological stomatognatic muscle relaxation as alternative treatments of temporomandibular joint dysfunction. Kraków: Monograph;2010.

2. Okeson J. Management of temporomandibular disorders and occlusion, $7^{\text {th }}$ edition. Elsevier: New York, USA;2013.

3. Trovato F, Orlando B, Bosco M. Occlusal features and masticatory muscles activity. A review of electromyographic studies. Stomatologija. 2009;11(1)29-31.

4. Castroflorio T, Falla D, Wang K, Svensson P, Farina D. Effect of experimental jaw-muscle pain on the spatial distribution of surface EMG activity of the human masseter muscle during tooth clenching. J Oral Rehabil. 2011;18:1365-1372. 
5. Kerstein R. Combining technologies: A computerized occlusal analysis system synchronized with a computerized electromyogeaphy system. Cranio. 2004;22(2)96-109.

6. Kim J, Shin J, Kim S, Kim C. Effect of two different units of botulinum toxin type a evaluated by computed tomography and electromyographic measurement of human masseter muscle. Plast Reconstr Surg. 2007;119(2):711-717.

7. Van der Bilt A, Abbink J, Fontijn-Tekamp F, Bosman F. Maximal bite forces and EMG during bilateral and unilateral clenching. J Oral Rehab. 2002;29:878-879.

8. Waltimo A, Kononen M. Maximal bite force and its association with signs and symptoms of craniomandibular disorders in young Finnish non - patients. Acta Odontol Scand. 1995;53(4):254-258.

9. Pihut M, Gierowski J. Psychological stress in the temporomandibular joint dysfunction own research. Protet Stomatol. 2003;LIII(5):261-269.

10. Chladek W. The mechanical modeling of selected states of the human mandible. Habilitation monograph. Gliwice: Politechnika Śląska; 2000.

11. Chladek W, Karasiński, Mol M, Lipski T, Kokot T. The use of computer simulation to evaluate the forces occurring during central occlusion. Protet Stomatol. 2003;LIII(4):196-202.

12. Pihut $M$. The influence of the uses of occlusal splint on the biomechanical conditions of chewing in the treatment of temporomandibular joint disorders. Monograph. Kraków; 2002.

13. Pihut M, Wiśniewska G, Majewski S. Evaluation of the effectiveness of relaxation of selected masticatory muscles under the influence of the use of occlusal splints using electromyographic studies. Czas Stomatol. 2007; LX(7):473-482.

14. Pihut M, Gierowski K, Ceranowicz P, Ferendiuk E. Psychoemotional background of the temporomandibular joint dysfunction and possible drug therapy. Lett Drug and Des Discov. 2015;12:766-770.

15. Fallah $\mathrm{H}$, Currimbhoy $\mathrm{S}$. Use of botulinum toxin $A$ for treatment of myofascial pain and dysfunction. J Oral Maxillofac Surg. 2012;70(5):1243-1245.

16. Velly A, Look J, Schiffman E, et al. The effect of fibromyalgia and widespread pain on the clinically significant temporomandibular muscle and joint pain disorders - a prospective 18-month cohort study. J Pain. 2010;11:1155-1164.

17. Margielewicz J. Numerical modeling in identifying biomechanical conditions of the stomatognathic system. Monograph. Zabrze; 2010.
18. Margielewicz J, Kijak E, Lipski T, Pihut M. Kosiewicz J, Lietz-Kijak D. Numerical modeling in identifying biomechanical conditions of the stomatognathic system. Monograph. Gliwice; 2012.

19. Nickel J, Spilker R, Iwasaki L, et al. Static and dynamic mechanics of the temporomandibular joint:plowing forces, joint load and tissue stress. Orthod Craniofac Res. 2009;12:159-167.

20. Reissmann D, John M, Schierz O, Wassell R. Functional and psychosocial impact related to specific temporomandibular disorders diagnoses. J Dent. 2007;35(8):643-650.

21. Miralles $R$, Zunino $P$, Santander $H$, Manns A. Influence of occlusal splint on bilateral anterior temporal EMG activity during swallowing of saliva in patients with craniomandibular dysfunction. Cranio. 1991;9(2):129-136.

22. Wassel R, Adams N, Kelly P. Treatment of temporomandibular disorders by stabilizing splints in general dental practice: Result after initial treatment. Br Dent J. 2004;10(197): 35-41.

23. Pihut M, Kazana P, Wiśniewska G, Łopuch P. Muscle exercises recommended in cases of functional disorders of the masticatory system. Implantoprotetyka. 2010; XI(2-3);38-44.

24. Żmudzki, J, Chladek, G, Kasperski, J. The influence of a complete lower denture destabilization on the pressure of the mucous membrane foundation. Acta Bioeng Biomech. 2012;14(3):67-73. doi: 10.5277/abb120309.

25. Fernandez $P$, at al. The region-dependent dynamic properties of porcine temporomandibular joint disc under unconfined compression. J Biomechanics. 2013;22(46):845-848.

26. Commisso MS, Martínez-Reina J, Mayo J, Domínguez J. Numerical simulation of a relaxation test designed to fit a quasi-linear viscoelastic model for temporomandibular joint discs. Proc Inst Mech Eng H. 2013;227(2):190-199.

27. Juran CM, Dolwick MF, McFetridge PS. Shear mechanics of the TMJ disc: Relationship to common clinical observations. J Dent Res. 2013;92(2):193-198.

28. Chladek, G, Barszczewska-Rybarek, I, Lukaszczyk, J. Developing the procedure of modifying the denture soft liner by silver nanoparticles. Acta Bioeng Biomech. 2012;14(1):23-29.

29. Miernik $M$, Więckiewicz $W$. The basic conservative treatment of temporomandibular joint anterior disc displacement without reduction-review. Adv Clin Exp Med. 2015;24:731-735. 